\title{
Commentary on "Do women runners report more pelvic floor symptoms than women in CrossFit? A cross-sectional survey" by Forner et al.
}

\author{
Marianne $\mathrm{Koch}^{1}$ \\ Received: 9 October 2020 / Accepted: 2 November 2020 / Published online: 16 November 2020 \\ (C) The International Urogynecological Association 2020
}

Increases in intra-abdominal pressure during physical exercise may negatively impact the pelvic floor and favor the development of pelvic floor dysfunction (PFD). Thus, practitioners may advise women to refrain from such activities in order to protect pelvic floor function. Through this cross-sectional survey, Forner et al. [1] aimed to understand whether heavyweight-lifting training programs, specifically $\mathrm{CrossFit} \odot$ training, foster the development of PFD in women.

To answer this question, a global online survey was set up inviting women who currently participated in either CrossFit@ training (including weight-lifting of $>15 \mathrm{~kg}$ ) or running (and no weight lifting $>15 \mathrm{~kg}$ ). Participating women were asked to submit weekly training frequency and length of time per session. Risk factors for PFD and PFD symptoms were collected (using the Pelvic Floor Distress Inventory (PFDI)-20). Analysis of data from 1,379 women (521 runners vs 858 CrossFit@ trainers) showed significantly higher overall PFDI scores in runners $(22.9$ vs $17.7 ; p<0.001)$; as well as a significantly higher Pelvic Organ Prolapse Distress Inventory (4.2 vs 0; $p<0.001$ ) and Colorectal-Anal Distress Inventory (6.3 vs 3.1; $p<0.001$; anal incontinence) scores in runners compared with women of the CrossFit $\odot$ group. No difference was found in Urinary Distress Inventory scores ( 8.3 vs $8.3 ; p=0.18$ ).

Marianne Koch

marianne.koch@meduniwien.ac.at

1 Department of Obstetrics and Gynecology, Medical University of Vienna, Vienna, Austria
Results from this study show a higher prevalence of PFD in female runners than in women who participate in CrossFit@ training, suggesting that $\mathrm{CrossFit} \odot$ training might not be more harmful to the pelvic floor than running. As the authors correctly state in the article, however, the study's design as a cross-sectional study does not allow conclusions on causalities to be drawn. The data collected did not include the overall time since the women participated in the respective sport (years? months? weeks?) either, and whether or not they already had PFD symptoms before the start of their training, or whether they will develop them later on in life. Thus, we should remain careful about counseling women on the safety of heavy-weight-lifting regarding pelvic floor dysfunction, as scientific data are not yet robust enough.

\section{Reference}

1. Forner LB, Beckman EM, Smith MD. Do women runners report more pelvic floor symptoms than women in CrossFit $($ ? A crosssectional survey. Int Urogynecol J. 2020. https://doi.org/10.1007/ s00192-020-04531-x

Publisher's note Springer Nature remains neutral with regard to jurisdictional claims in published maps and institutional affiliations. 